\title{
A Wrist-type Multi-physiological Signal Acquisition Device
}

\author{
Xinwei Zhou ${ }^{1, a}$, Xiaoyong $\mathrm{Ji}^{1, \mathrm{~b}}$ and Xinyu Chai ${ }^{1, \mathrm{c}}$ \\ ${ }^{1}$ School of Biomedical Engineering, Shanghai Jiao Tong University, Shanghai 200240, China \\ a31zhouxinwei@sjtu.edu.cn, bxyjisjtu@163.com, cxychai@sjtu.edu.cn
}

\begin{abstract}
Keywords: Electrodermal activity, Heart rate, Ambient temperature, Motion detection, Wrist-type Abstract. Affective Computing is computing that relates to, arises from, or deliberately influences emotion or other affective phenomena. Emotion recognition based on affective computing is an important part of affective computing. Electrodermal activity signal and heart rate signal are important parameters of emotion recognition. In order to achieve these physiological parameters in a long-term and real time condition, a multi-physiologic signal acquisition and storage device is designed. The micro-control unit of the device adopts the low-power processing chip MSP430F5529 produced by Texas Instruments, and integrates electrodermal activity, heart rate, ambient temperature, and motion detection, which can realize wearable multi-parameter collaborative monitoring system design, providing a precise and reliable technology platform.
\end{abstract}

\section{Introduction}

In the daily life, work, communication, handling affairs, and decision-making, people's emotional ability plays just as important role as the normal rational thinking and logical reasoning ability. Rosalind Picard's 1995 paper on affective computing defines that affective computing is computing that relates to, arises from, or deliberately influences emotion or other affective phenomena [1]. In a sense, emotion is the external manifestation of affective and affective is the essence of emotion[2]. Therefore, emotion recognition is one of the most important part of affective computing.

Physiological signal is only changed by the autonomic nervous system and endocrine system and it can't be controlled by subjective. So, using physiological signal is more objective. Electrodermal activity (EDA) is the property of the human body that causes continuous variation in the electrical characteristics of the skin. Skin resistance changes with the state of sweat glands in the skin. Sweating is controlled by the sympathetic nervous system [3]. Heart rate (HR) is the speed of the heartbeat measured by the number of contractions of the heart per minute and is related with autonomic nervous system [4]. It implies EDA signal and HR signal contain extremely rich emotional information, and can reflect emotional changes [5]. EDA and HR are often affected by three factors which are human emotion, ambient temperature, and motion state. Combined with the correction of ambient temperature and motion detection data, emotion can be accurately recognized based on EDA signal and HR signal. At present, wearable multi-physiological signal acquisition devices are generally large, expensive and so on.

The purpose of this research is to design a wrist-type multi-physiological signal acquisition device that can be comfortably worn for a long time and be inexpensive and reliable. The device can be used to collect long-term precise data, including EDA, HR, ambient temperature and motion state which are stored in microSD card for using afterward emotion recognition.

\section{System description}

Combined with the requirements of non-invasive, small size, long-term, and real-time recording data for emotional recognition, the wrist-type multi-physiological signal acquisition device is based on low-power processing chip MSP430(F5529) [6]. The device is mainly divided into eight modules, including the power module, the EDA module, the HR module, the motion detection module, the ambient temperature measurement module, the processing module, the storage module, and the programming module. EDA signal is sampled at $50 \mathrm{~Hz}$ and then is transformed to 12-bit digital signal through the ADC12 of MSP430. ADT7420(Low power consumption, $4 \mathrm{~mm} \times 4 \mathrm{~mm}$ 
[7]) is used to collect ambient temperature data and it samples at $1 \mathrm{~Hz}$ for energy saving. MMA8452Q (3 mm x $3 \mathrm{~mm} \times 1 \mathrm{~mm}$ [8]) is used to collect motion detection data and it samples at $50 \mathrm{~Hz}$. They are both communicated with MSP430 via the I2C protocol. FAT file system module suitable for micro embedded systems is transplanted into MSP430 in order to communicate with microSD card through Serial Peripheral Interface (SPI). As the device needs to store data for a long time, it uses an 8GB microSD card to save the signals. The whole software of the device is built on Code Composer Studio (CCS). The system block diagram illustrated in Fig. 1.



Fig. 1: Overview of wrist-type multi-physiological signal acquisition device system architecture

User comfort is a major consideration in the design of any wearable device intended for long-term and continuous use. In view of this, the device integrates the main modules on a $32 \mathrm{~mm} \mathrm{x}$ $50 \mathrm{~mm}$ circuit board (see Fig. 2(a)). In addition, up to now, there is no accepted standard on the location of $\mathrm{EDA} \mathrm{Ag} / \mathrm{AgCl}$ electrodes [9]. The measuring electrodes are usually placed on the surface of the palm, the most popular sites being the medial and distal phalanges of the fingers, and the thenar and hypothenar eminences. However, hands are the most frequently used parts in people's daily life. If electrodes are placed on the hands, it will interfere with people's daily life. According to Picard's research, the distal end of the forearm can acquire effective EDA data too [10], so we also choose this recording site (see in Fig. 2(b)). Thus, the circuit board is fixed on the bracer and is worn on the wrist (see in Fig. 2(c)).

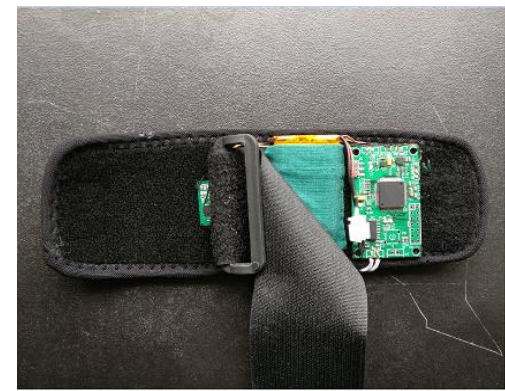

(a)

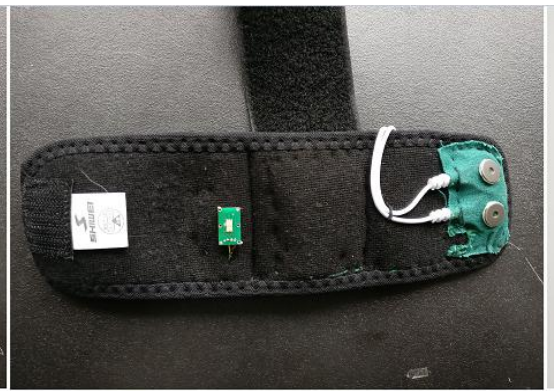

(b)

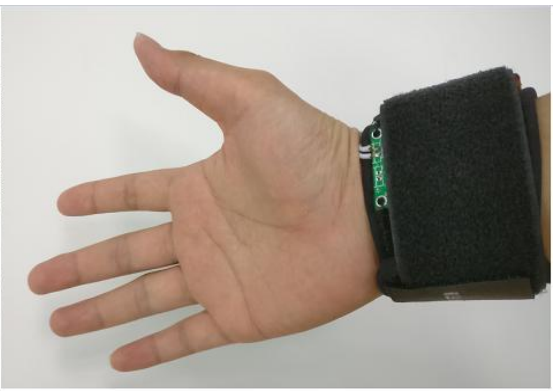

(c)

Fig. 2: (a) The front of the device with the main PCB, ambient temperature measurement PCB and Li-Poly battery; (b) The back of the device with HR PCB and $\mathrm{Ag} / \mathrm{AgCl}$ electrodes; (c) device can be worn comfortably on the wrist for long periods of time and during daily activities. 


\section{Applications}

In order to provide accurate data for emotion recognition research, it is necessary to verify the accuracy of the data collected by the device.

The EDA signal data measures the skin resistance, so the accuracy of the device is able to be analyzed by measuring the resistance of a known one. The resistance of EDA signal distribution range is large, generally in the $100 \mathrm{~K}-2.5 \mathrm{M}$. Table 1 shows that compared measuring the skin resistance value with the known resistance by the Agilent digit multimeter, and the percentage error between the actual value and measurement value is below $1 \%$. The result indicates an accurate estimation of EDA signal from our device.

Table 1. the actual resistance value and measurement value

\begin{tabular}{|c|c|c|}
\hline $\begin{array}{c}\text { Resistance } \\
(\mathrm{k} \Omega)\end{array}$ & $\begin{array}{c}\text { Measurement } \\
(\mathrm{k} \Omega)\end{array}$ & $\begin{array}{c}\text { Percentage } \\
\text { Error }\end{array}$ \\
\hline 102.08 & 101.78 & $-0.29 \%$ \\
\hline 147.22 & 147.52 & $0.20 \%$ \\
\hline 198.57 & 198.63 & $0.03 \%$ \\
\hline 250.88 & 250.44 & $-0.17 \%$ \\
\hline 297.52 & 297.44 & $-0.03 \%$ \\
\hline 355.01 & 355.59 & $0.16 \%$ \\
\hline 399.67 & 399.41 & $-0.05 \%$ \\
\hline 448.52 & 449.68 & $0.26 \%$ \\
\hline 493.40 & 494.22 & $0.17 \%$ \\
\hline 552.50 & 552.77 & $0.05 \%$ \\
\hline 657.27 & 659.37 & $0.32 \%$ \\
\hline 747.42 & 749.77 & $0.31 \%$ \\
\hline 848.98 & 852.58 & $0.42 \%$ \\
\hline 953.21 & 956.94 & $0.39 \%$ \\
\hline 1015.02 & 1019.70 & $0.46 \%$ \\
\hline 1514.20 & 1525.74 & $0.76 \%$ \\
\hline 2025.30 & 2034.97 & $0.48 \%$ \\
\hline
\end{tabular}

The HR sensor is a highly integrated pulse meter module using photoplethysmograph (PPG) reflective method to measure human heart beat. The heart rate sensor of our device is placed on the wrist. Five healthy subjects are measured heart rate for 15 seconds by our device and Omron Electronic Blood Pressure Monitor measures their heart rate at the same time on the other hand. The result of the experiment shows our device can provide accurate heart rate data in real time and the error is less than 3 beat $/ \mathrm{min}$.

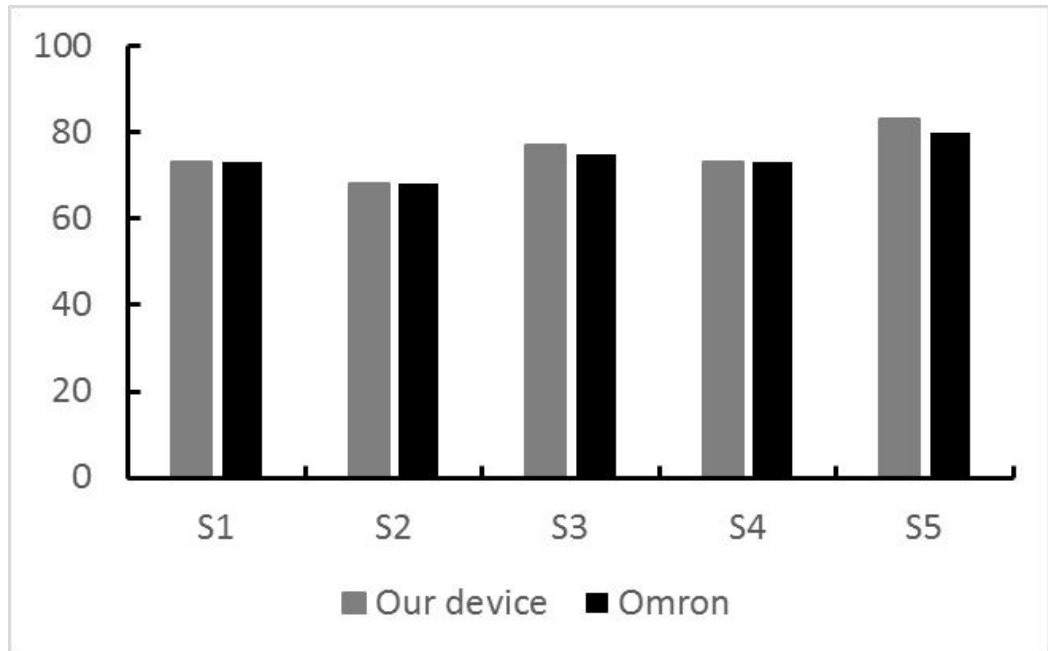

Fig. 3: HR data on Our device HR data and Omron 


\section{Conclusions}

A new wrist-type multi-physiological signal detection and analysis device based on multiple sensors has been developed and is currently under test. The device is a comfortable-wear, special purpose device, which is designed to picks up bio-signals usable for recognizing user's emotion. The first positive results obtained from our device shows that it can provide precise and reliable data and the manufacturing cost of the device is low. Future work includes using these data to build our own emotional database and recognize users' emotion.

\section{Acknowledgements}

This work was financially supported by The National Basic Research Program of China (973 Program, 2011CB707503); The National Natural Science Foundation of China (61273368, 61472247, 91120304); National High Technology Research and Development Program of China (863 Program, 2009AA04Z326).

\section{References}

[1] Picard R W. Affective computing[J]. 1995.

[2] Xiaowei Liu. Affective education[M]. East China Normal University Press. 2007.

[3] Bartholomew E F, Martini F, Ober W B. Essentials of anatomy \& Physiology[M]. Benjamin Cummings, 2007.

[4] Agrafioti F, Hatzinakos D, Anderson A K. ECG pattern analysis for emotion detection[J]. IEEE Transactions on Affective Computing, 2012, 3(1): 102-115.

[5] Kreibig S D. Autonomic nervous system activity in emotion: A review[J]. Biological psychology, 2010, 84(3): 394-421.

[6] Instruments T. MSP430F5529 datasheet[J]. 2011.

[7] PrB R. Preliminary Technical Data ADT7420[J].

[8] MMA8452Q X. 3-Axis, 12-Bit/8-Bit Digital Accelerometer[J]. 2013-10.

[9] W. Boucsein, Electrodermal Activity. New York: Plenum, 1992.

[10] Poh M Z, Swenson N C, Picard R W. A wearable sensor for unobtrusive, long-term assessment of electrodermal activity[J]. IEEE transactions on Biomedical engineering, 2010, 57(5): $1243-1252$. 